\title{
Restitution numérique, réappropriations amérindiennes. Introduction à la seconde partie
}

\section{Valentina Vapnarsky et Camille Noûs}

\section{(C) OpenEdition}

1 Journals

Édition électronique

URL : https://journals.openedition.org/jsa/19495

DOI : 10.4000/jsa. 19495

ISSN : $1957-7842$

Traduction(s) :

Digital repatriation, Amerindian reappropriations. Introduction to Part Two - URL : https://

journals.openedition.org/jsa/19794 [en]

Éditeur

Société des américanistes

Édition imprimée

Date de publication : 30 septembre 2021

Pagination : 113-125

ISSN : 0037-9174

Référence électronique

Valentina Vapnarsky et Camille Noûs, "Restitution numérique, réappropriations amérindiennes. Introduction à la seconde partie », Journal de la Société des américanistes [En ligne], 107-1 | 2021, mis en ligne le 30 septembre 2021, consulté le 05 septembre 2022. URL : http://journals.openedition.org/ jsa/19495; DOl : https://doi.org/10.4000/jsa.19495

Ce document a été généré automatiquement le 5 septembre 2022.

Tous droits réservés 


\title{
Restitution numérique, réappropriations amérindiennes. Introduction à la seconde partie
}

\author{
Valentina Vapnarsky et Camille Noûs
}

Believing, with Max Weber, that man is an animal suspended in webs of significance he himself has spun, I take culture to be those webs, and the analysis of it to

be therefore not an experimental science in search of

law but an interpretive one in search of meaning.

(Geertz 1973, p. 5)

1 Le passage bien connu que nous donnons en exergue définit la culture comme une toile de significations tissée par les humains pour mieux s'y suspendre et, peut-on supposer, y attendre confortablement leurs proies en position panoptique. Déjà frappante au(x) siècle(s) précédent(s), l'image l'est d'autant plus à l'heure du numérique, où l'association des notions de «toile» et de "culture » relève désormais de la cyber-évidence. Mais qu'advient-il lorsque votre toile a été tissée par d'autres, et qu'on risque de s'y trouver bien plutôt englué que suspendu? Le cybermonde est-il vraiment ce qu'il prétend être, ouvert et accessible à tous, ou ne serait-il qu'un avatar sournois de l'impérialisme culturel ? Comme le notait James Maffie (2009) dans un article méditant sur le devenir des savoirs autochtones: "In the end, we have the Gatling gun [mitrailleuse], and they have not». Que la force de frappe se comptabilise aujourd'hui en termes de capacité à structurer les métadonnées plutôt qu'à faire crépiter une arme automatique ne change pas grand-chose à l'affaire.

2 On a souvent relevé que la restitution numérique a ceci de paradoxal qu'elle ne peut restituer que ce qui avait été préalablement aliéné par l'acte même de collecte, érodé par le passage du temps, et reconfiguré par le contexte encadrant son retour. Comme développé dans l'introduction de la première partie de ce dossier (Vapnarsky 2020), conserver, c'est nécessairement transformer. Dès lors, ce qui revient est nécessairement pourvu d'un statut hybride et n'aura de sens, pour les communautés d'origine, qu'au prix 
d'ardentes tractations intra- et interculturelles. Retour aux sources, certes, mais l'on ne se baigne jamais deux fois dans le même fleuve. En outre, comme l'a très justement souligné Eric Jolly (2008), les fonds d'archives doivent être compris comme des points de vue successifs sur une société donnée et non comme dépositaires de traditions telles qu'elles ont pu exister. Ils résultent de sélections en cascade, dépendantes de l'angle d'observation adopté, des contraintes techniques des appareils d'enregistrement, et des choix d'archivage et de mise à disposition. Prises de son, de vue ou de notes sont relatives à un point de vue, à un moment historique et politique donné. Ils correspondent à des objectifs de recherche spécifiques (Naepels 2008) et peuvent dès lors décevoir les membres des communautés sources qui se les voient restituer (Christen 2009).

3 Bon nombre d'acteurs institutionnels semblent avoir bien compris l'importance cruciale de la co-construction du sens dans la valorisation des fonds d'archive que permet le numérique. Notre époque est clairement marquée par une sensibilisation accrue à l'éthique de la concertation et à ce qu'on a pu appeler les processus de légitimation des collaborations (Delaître et Robert 2019). Désormais, comme le soulignait Peers (2010) à propos de la collaboration entre conservateurs de musée et populations autochtones, l'accent est mis sur l'importance d'un dialogue qui prenne en compte:

The shift from the assumption that museums exist to house relics of dying cultures to seeing museums as material archives, resources for living cultures; the shift from museum staff being authorities on Indigenous cultures to acknowledging that Indigenous people are the authorities on their own cultures; the shift from thinking about museum objects as things, to thinking of them as potentially animate, and as embodying sets of relationships; the shift from museums working in isolation from source communities to working in partnership with them. (Peers 2010, p. 187)

4 Il faut bien sûr se réjouir de cette évolution ainsi que de la tendance, de plus en plus répandue, d'atténuer autant que possible le caractère unilatéral des projets de sciences humaines ou d'ingénierie culturelle (Athias 2018; Hoffmann et Noack 2016; Kraus 2016; Pierrebourg à paraître; Van Velthem et al. 2017). Plutôt que de " restitution », tant pour les musées que pour les archives, ne vaut-il pas mieux parler de «partage » (Jolly 2008)? Mais ne nous leurrons pas pour autant: «collaboratif» n'est pas, loin s'en faut, synonyme de strictement paritaire. L'asymétrie va parfois se nicher jusque dans les projets explicitement destinés à s'en démarquer, où il arrive par exemple que le nom des contributeurs autochtones se trouve relégué dans une dénomination générique, n'apparaissant plus qu'en filigrane, dans l'anonymat d'un codicille scripturaire. Plus contraignants, les fondements des infrastructures patrimoniales - depuis les législations et exigences de la conservation, la structuration des bases de données, l'herméticité aux langues dites " peu dotées ${ }^{1}$ ", jusqu'aux arcanes documentaires de l'accès aux matériaux et celles technocratiques de l'accès aux financements - rend difficile, voire parfois impossible, l'intégration des perspectives et des initiatives des populations autochtones. La bonne volonté ne suffit pas toujours.

5 L'introduction de la première partie de ce dossier se terminait sur la suggestion optimiste d'une compatibilité remarquable entre les potentialités du numérique et les modalités de circulation et de reconfiguration du savoir dans les sociétés amérindiennes. Peut-être en va-t-il de même pour la plupart des sociétés à tradition orale ? Comparé aux 
médias traditionnels, le numérique affiche plus de flexibilité et de polyvalence, ainsi qu'une meilleure capacité à rendre compte de la dimension multimodale des matériaux et performances captés, ou capturés. En outre, dans des sociétés autochtones très ouvertes sur l'extérieur et perméables aux rencontres interculturelles, circulation, mobilité et plasticité ont toujours existé. Or, comme le soulignait Achille Mbembe : " cette souplesse et cette aptitude à l'innovation constante, à l'extension du possible, c'est aussi l'esprit d'Internet, [...] l'esprit du numérique » (Mbembe 2017, p. 204). Lorsqu'il affirmait que "le pouvoir des technologies numériques vient de leur capacité à être détachées de leur ensemble originel pour être incorporées à une autre matrice culturelle» (ibid., p. 199), le philosophe pensait certes au continent africain, mais le constat vaut aussi pour les Amériques. Et on ne peut évidemment qu'être séduits par cette image de civilisations extra-occidentales "déjà numériques avant le numérique " (ibid, p. 204), utilisant Internet comme «un grenier portable pour bien des connaissances » (ibid., p. 198).

6 Une certaine prudence semble toutefois s'imposer. L'accès au réseau et la capacité de s'y retrouver restent compliqués dans certaines parties du monde, et sans doute ne suffit-il pas de les rendre "virtuellement" accessibles en ligne pour décoloniser les bases de données. Le "virtuel », par définition, ne peut advenir qu'en cessant, précisément, de l'être, et pour cela des moyens bien concrets sont nécessaires. Pour circuler sur la "toile», il faut du réseau, au sens informatique, mais sans doute aussi en termes de relations sociales (Glinka, Meier et Dörk 2015). Il faut du hardware, du software, du débit, mais aussi des passeurs, des entremetteurs culturels et de la formation, initiale et continue... Souvent, les données étaient déjà disponibles bien avant que quiconque ne s'avise d'en signaler l'existence et de les rendre accessibles aux communautés dont elles proviennent. Elles n'étaient toutefois accessibles qu'aux geeks surdiplômés, surfeurs invétérés, anglophones de préférence ; une engeance qui compte peu de représentants dans les villages reculés des Andes ou de Patagonie. Ce qu'on croyait mégaphone s'avère parfois sourdine, pour reprendre une métaphore de Gibson et Kahn (2016).

7 Ainsi, le portail Watau ${ }^{2}$ - dont une présentation dans les communautés de Guyane sert de couverture au dossier - a été élaboré, entre autres raisons, pour répondre aux préoccupations d'un groupe d'érudits wayana qui s'interrogeaient sur le devenir des clichés, des enregistrements et des autres artefacts que plusieurs générations de chercheurs européens étaient venus chercher chez eux. Où sont donc tous ces matériaux ? Pourquoi les voulaient-ils? Qu'en a-t-il été fait ? Comment y avoir accès? Les réponses étaient certes disponibles dans les catalogues des musées, les bibliothèques universitaires, les archives, les photothèques, etc., mais il s'agit là d'autant d'endroits où - comme le disait Mataliwa Kuliyaman avec son humour habituel - l'on ne peut guère accéder avec nos pirogues ${ }^{3}$. Quant aux ressources en ligne, sans doute n'est-il pas indifférent que le néologisme retenu par les Wayana pour se référer au web soit ëwa, autrement dit un terme désignant la corde merveilleuse qui permet aux chamanes (et à eux seuls) d'accéder aux différentes strates du cosmos ${ }^{4}$. Les autres resteront ils englués sur la toile?

8 Au sein du présent dossier, l'article de Vincent Hirtzel fournit une autre bonne illustration de ces nombreux cas où la restitution « on line» - pourtant partie intégrante du projet qu'il décrit - ne suffisait pas. Ses interlocuteurs yurakaré ont tenu à récupérer les données sous forme de disques durs, mieux à même de "réifier» leur culture, de lui donner une incarnation bien concrète, susceptible d'être exhibée lors de réunions 
publiques autrement plus fréquentées par leurs proches que les sites web. Le récit des obstacles rencontrés lors de l'élaboration collective d'un portail numérique interculturel présenté par Andrea Scholz et Mirĩgõ-Diana Guzmán Ocampo témoigne également des difficultés inhérentes à la dimension excessivement technique, pré-structurée et onéreuse de telles entreprises. Des retombées positives inattendues peuvent cependant en résulter, comme la mise en place d'ateliers de tressage de vannerie en lieu et place d'ateliers de remplissage de fiches de métadonnées sur les paniers. Peut-être faut-il en déduire (mais c'est une évidence) que la restitution n'est pas une fin en soi, destinée à la seule contemplation, mais une étape dans un processus nettement plus complexe. L'exemple des Xetá du sud du Brésil, décrit par Edilene Coffaci de Lima, Lilianny Rodriguez Barreto dos Passos et Rafael Pacheco montre bien qu'à partir de ce qui est "retourné », on peut aller jusqu'à se reconstituer une identité collective et tenter de récupérer son territoire ancestral. Exister, en somme.

9 Le symposium à l'origine des articles publiés dans ce dossier spécial sur la restitution numérique s'intitulait : Digital repatriation of traditional indigenous knowledge: a threat or an asset for its transmission? (La restitución digital de saberes indígenas tradicionales: ¿un recurso o una amenaza para su transmisión?). Les présentations qui y ont été faites, ainsi que les publications subséquentes, montrent toutefois que nous manquons encore de recul pour envisager cette question de manière approfondie. Plutôt que l'impact des nouvelles technologies sur la transmission des savoirs, la plupart des communications, comme par ailleurs l'essentiel de la littérature spécialisée que nous avons consultée, se sont d'abord attachées à simplement décrire les initiatives de restitution. Et ce, en mettant surtout l'accent sur les effets positifs du partage numérique (Powell 2016), bien que le doute soit de mise (Göbel et Chicote 2017). Pour le dire autrement, comme poussés par le besoin de se justifier, on s'est jusqu'ici beaucoup concentrés sur les aspects bénéfiques de ces entreprises, et les bonnes intentions qui ont incité à s'y engager. Plus rares sont les témoignages de situations où les entreprises de restitution aboutissent à une impasse. Par exemple, à une restriction des droits d'accès au contenu, non seulement pour les " étrangers", mais aussi pour certains membres du groupe, ceux-ci pouvant s'en voir privés pour des raisons découlant d'enjeux de politique identitaire ou de régimes épistémiques locaux (Cantz 2018)5. Les échecs et les effets pervers ne sont pourtant pas rares, comme en attestent certains des cas analysés dans l'ensemble de textes proposés ici. De même que les projets de développement, les projets culturels sont eux aussi exposés aux malentendus de tout ordre et à ce qu'on a pu appeler "the clash of cosmographies» (Wright, Kapfhammer et Wiik 2012).

Une partie des quiproquos provient sans doute de ce que le statut accordé à ce qui est « restitué » peut varier selon la perspective adoptée (Shepard et al. 2017). Ce qui ressort de la «culture» du point de vue des institutions patrimoniales, mais aussi des anthropologues, peut par exemple relever plutôt de la «mémoire familiale » dans l'optique des communautés sources. Dans d'autres cas, les récipiendaires n'ont qu'un lien fort ténu, voire totalement fantasmatique, avec des pratiques imputées à des aïeux autoproclamés. C'est souvent là que surviennent des conflits, comme il ressort des analyses d'Augustat (2020) sur les tentatives, par les Pataxó du Brésil, de reconstruire leur patrimoine culturel perdu à partir d'éléments produits par les ancêtres d'autres Amérindiens et préservés dans les réserves de musées allemands ${ }^{6}$. Alors même que toute 
l'anthropologie de ces dernières décennies s'interroge sur la complexité (et, parfois, sur la pertinence) de la notion de culture, on "restitue " à tour de bras dans l'optique de "revitalisation linguistique et culturelle», sans trop se préoccuper de ce qu'il faut mettre derrière ces termes.

Transposés en megabytes, les ektachromes, les plaques de verre, les manuscrits, les incunables, les cassettes, les cylindres de cire et autres objets jadis trop fragiles pour circuler peuvent certes, enfin, sortir de leur(s) réserve(s). Guidés par des anthropologues ou des conservateurs altruistes, leurs avatars numériques peuvent dès lors retrouver le chemin des communautés sources, sans risquer pour autant de disparaitre à tout jamais. D'aucuns s'offusqueront qu'on ne restitue que des «reproductions " mais, à bien des égards, la reproduction n'est-elle pas l'essence même de la vie ${ }^{7}$ ? Pourtant, quoique simples en apparence, les opérations de "restitution" entraînent souvent des effets sociaux inattendus, complexes, parfois troublants, toujours fascinants. On l'a vu, dans la première partie de ce dossier, à partir des expériences relatées par Clarice Cohn, Maria Luisa Lucas, Jean Langdon et Margarita Valdovinos respectivement chez les Kayapo, les Bora, les Siona et les Cora (voir JSA, 106-2). Les articles présentés ci-dessous l'illustreront aussi, de manière complémentaire.

Les quatre textes proposés dans la première partie de ce numéro spécial mettaient l'accent sur des initiatives de restitution individuelles, autrement dit des situations où les chercheurs (en l'occurrence, chercheuses) souhaitaient partager avec leurs interlocutrices et interlocuteurs amérindiens des sources concernant leur passé, leurs traditions et leur culture. Elles le faisaient cependant en endossant essentiellement un rôle d'intermédiaire, rapportant des images, des textes ou des sons qui provenaient parfois de leurs collections personnelles, mais qui avaient aussi souvent été collectés par d'autres (parfois plusieurs décennies plus tôt) et qui, surtout, n'étaient pas initialement destinés à la restitution. Les trois articles intégrant ce second volet du dossier ont ceci en commun qu'ils illustrent plutôt des dispositifs liés à des programmes institutionnels, impliquant des formes de restitution numérique, soit comme condition de collecte, soit comme une fin en soi. Ces projets intègrent souvent une pluralité d'acteurs et, parfois, disposent de budgets conséquents, notamment dans le cadre d'ambitieuses entreprises collectives portées par des institutions muséographiques ou universitaires, et visant à la constitution de bases de données ou de plateformes numériques.

Dans la continuité des précédents articles, ceux de la présente livraison mettent tous en exergue la variété des réappropriations personnelles, identitaires et politiques des objets restitués virtuellement - c'est-à-dire sous forme numérique et parfois encore potentiellement. L'exemple dramatique des Xetá, décrit dans l'article de Edilene Coffaci de Lima, Lilianny Rodriguez Barreto dos Passos et Rafael Pacheco, en représente un cas extrême. En effet, les quelques survivants de ce peuple amazonien décimé sont devenus tributaires des archives pour justifier de leur propre existence, hier et aujourd'hui, en tant qu'ethnie. Les deux autres articles suivants illustrent les nouvelles configurations qu'induit la restitution. D'une part, sur le terrain, celle-ci est devenue une composante intégrante de la collecte de matériaux ethnographiques ou linguistiques. Superposant des processus et des temporalités autrefois disjoints, elle transforme la façon dont les participants locaux investissent leur relation à l'enquête, autant dans sa portée 
sociopolitique et historique que dans ses resignifications intimes et personnelles. C'est ce que nous montre l'article de Vincent Hirtzel sur un programme DoBes mené chez les Yurakaré de Bolivie. D'autre part, le processus de restitution est également entré dans l'arène du musée, le mode numérique fournissant une alternative séduisante au retour matériel des objets. Au-delà de l'accès aux objets, en consultation privilégiée sur place ou en reproduction négociée à distance, le dialogue qui s'établit entre les représentants des groupes ethnographiés et les conservateurs ou documentalistes conduit à revoir les formes de présentation, de classification et de documentation des matériaux (pour ne rien dire de la question de leur conservation et éventuelle restauration). Cependant, la flexibilité promise par les infrastructures numériques s'avère vite assez illusoire, ces dernières agissant plutôt comme des étaux qui contraignent fortement, voire interdisent, l'intégration des perspectives autochtones, quelles que soient les bonnes intentions des concepteurs des bases de données patrimoniales. Se libérer de ces contraintes, les détourner, et peut-être in fine les contrer, implique le recours à des procédés très artisanaux. Il faut parfois retrouver le papier et le crayon, l'esprit et la main de la fabrication, tout autant qu'obtenir l'appui d'institutions puissantes prêtes à financer des projets d'ingénierie informatique coûteux en temps et en moyens, alors même que les communautés visées sont souvent peu visibles et très " minoritaires". L'article d'Andrea Scholz et Mirĩgõ-Diana Guzmán Ocampo, écrit à deux voix, du point de vue du musée, l'Ethnologisches Museum de Berlin, et de celui des communautés locales, celles de la région du Vaupés en Colombie, en fournit une illustration exemplaire.

14 L'article co-signé par Edilene Coffaci de Lima, Lilianny Rodriguez Barreto dos Passos et Rafael Pacheco, présente le cas à la fois tragique et remarquable d'un groupe dont l'existence même semble indissociablement liée aux projets de documentation (muséographiques, pour l'essentiel). À certains égards, on peut même dire que les Xetá (Paraná, Brésil) sont eux-mêmes des archives. Des archives vivantes, puisque depuis plusieurs générations, ils semblent n'exister que pour donner des témoignages de leur ancienne vie. Les rares Xetá ayant survécu aux massacres concomitants à la collecte des pièces et captations audiovisuelles qui, pour les ethnologues de cette époque, témoignaient des " derniers Indiens de l'âge de pierre », ont été dispersés et empêchés de vivre ensemble. Spoliés des moyens de survie en tant que groupe politiquement homogène, ils comptent sur les archives pour retrouver une cohésion sociale et une légitimité pour demander des terres où retrouver une " culture » (au sens politique). Les archives numériques et les copies fournies par divers anthropologues offrent une matérialité paradoxale à la virtuelle existence ethnique du groupe. Les disques durs et les clefs USB sont conservés et consultés avec engouement et émotion. Les Xetá se les sont répartis selon une logique de division du travail mémoriel : le frère aîné se spécialise dans les histoires, le cadet dans les chants et la langue, tandis que sur les sœurs reposent la récupération de certaines techniques artisanales. Les maisons deviennent dès lors des sortes de salles d'un musée ethnographique vivant, vécu et monitoré par ceux qui en sont autant les objets que les sujets. Les archives numériques acquièrent quant à elles un statut de reliques, attestant d'un passé à jamais perdu, mais sans lequel les Xetá ne peuvent plus concevoir leur devenir.

15 Le second texte, signé par Vincent Hirtzel, relate son expérience dans un projet DoBeS mené conjointement avec des linguistes et des locuteurs du yurakaré, langue amazonienne parlée dans le piémont andin, en Bolivie. Destiné à la constitution de corpus linguistiques, le financement de ce projet a eu pour condition de prévoir, dès sa 
conception, la restitution des matériaux enregistrés. Cette condition, associée à celle de dimension collaborative, figure désormais dans les appels de la plupart des grands programmes de financement de documentation linguistique ou culturelle. Elle bouleverse les relations entre collecte et restitution. En effet, alors que celles-ci constituaient deux phases nettement séparées, tant en termes temporels que s'agissant des acteurs impliqués, les deux processus se trouvent aujourd'hui intégrés d'emblée dans une même démarche, la restitution s'accomplissant (ou à tout le moins se préparant) à mesure même que l'objet se construit. Cependant, si collecte et restitution sont désormais indissociablement liées, le rapport entre les deux n'en demeure pas moins complexe. Hirtzel souligne clairement l'importance, pour saisir les enjeux de la réception et des circuits locaux des copies, de la prise en compte du contexte politique, ainsi que de ce qu'il appelle les «régimes de culture» et du rapport à la patrimonialisation ayant cours à l'échelle nationale et régionale. Pour le dire autrement, il montre qu'un projet DoBeS sera reçu et approprié localement très différemment selon qu'il ait été mené dans les groupes du Haut Xingu au Brésil ou en Bolivie chez les Yurakaré.

Hirtzel s'interroge également sur les motivations individuelles des partenaires autochtones qui viennent spontanément offrir leur collaboration dans le cadre de tels projets. Entre situation d'enregistrement et perspective de restitution, ces projets se trouvent investis comme dispositifs dialogiques et canal de communication, mais avec ceci de particulier que le destinataire n'est pas forcément clairement identifié. La personne enregistrée s'adresse-t-elle avant tout à l'ethnologue ou au linguiste, à ellemême, à ses descendants (par anticipation), à sa communauté, à des Yurakaré inconnus, ou aux étrangers en général ? Et pourquoi avoir recours à l'autobiographie, choisir de relater des vécus très personnels plutôt que de se concentrer sur des récits à portée plus générale, moins tributaires des aléas du destin individuel et à ce titre plus calibrés, du moins du point de vue des collecteurs, pour être représentatifs d'une culture partagée (et à partager)?

17 Le troisième article, co-signé par Andrea Scholz et Mirĩgõ-Diana Guzmán Ocampo illustre remarquablement les enjeux, mais aussi les difficultés et les frustrations, inhérents à la dimension collaborative des projets de restitution numérique hébergés par les musées et les grandes institutions patrimoniales. Le projet «Compartir saberes » croisait les visées décoloniales du musée et celles des communautés sources. Le musée souhaitait ouvrir ses bases aux contributions des descendants des communautés du Nord-Ouest amazonien où avaient été collectés les objets, afin d'inclure les perspectives autochtones dans les discours muséographiques sur leur collection. Les communautés locales, quant à elles, étaient tout d'abord intéressées par l'obtention d'un accès à ces matériaux distants. Prises au jeu de produire, pour les musées, une version des réseaux de significations au sein desquels elles concevaient les objets collectés, la démarche à laquelle elles étaient invitées à participer s'avéra jonchée d'obstacles, depuis le "simple" accès au réseau internet, jusqu'à la possibilité d'intégration des multiples connexions qui définissaient les objets pour elles. Forcées dans un système de hiérarchie classificatoire imposée par les bases en ligne, ces connexions aboutissaient à une vision à l'opposé de celle du musée. Pour les communautés, il ne s'agissait pas de rendre compte d'un objet mais de "la vie des objets ", à savoir de leurs parcours et usages et de leurs multiples recontextualisations impliquant, par exemple, la relation à des ancrages géographiques divers, qu'ils concernent leur origine mythique, leur production, incorporation, utilisation, conservation, réutilisation... Il s'agissait de défaire le modèle 
d'univocité qui prévaut aux classifications muséales en (et hors) ligne : reconnaitre le lien d'un objet à différents groupes ethniques, intégrer le multilinguisme, tenir compte de la hiérarchisation locale des groupes et de la codification des droits d'accès aux savoirs, etc. Mais comme le révèle l'article, les bases de données patrimoniales habituelles ont ce "défaut de naissance » qui conduit à des exclusions en série. Toute initiative visant à introduire des logiques autres rebondit contre une paroi inflexible de code. Contre ce système que d'aucuns - comme nous - nommeraient pervers, il ne semble $\mathrm{y}$ avoir qu'une solution. Repartir à zéro. Reboot.

\section{BIBLIOGRAPHIE}

\section{ATHIAS Renato}

2018 « Museums, ethnographic collections, and virtual repatriation: new issues for an old debate ", in João Pacheco de Oliveira et Rita de Cássia Melo Santos (dir.), De acervos coloniais aos museus indígenas: formas de protagonismo e de construção da ilusão museal, Universidade Federal da Paraíba (UFPB), João Pessoa, p. 337-364.

\section{AUGUSTAT Claudia}

2020 " A play in the field of words: from material culture to/and cultural heritage ", Indiana, 37 (2), p. 237-245.

CAMARgo Éliane, Veronica HOLGUIN LEW, Sara TANDAR et Équipe Wayana et Apalaï [sic] 2020 « L'Amazonie amérindienne dans l'ère du numérique : le portail multilingue WATAU », Patrimoines du Sud [en ligne], 12, http://journals.openedition.org/pds/5127, consulté

le $10 / 06 / 2021$. DOI : $10.4000 /$ pds.5127

CAMARGo Éliane et Tapinkili [ANAIMAN]

2020 Wajana omi jatëku. Etapomi Hapon. Wayana [sic]-palasisi. Wayana au cœur des mots. Dictionnaire analogique bilingue Wayana-français, Ipe éditions/CTHS, Paris.

CANTZ Hatje

2018 Secrecy. Who's allowed to know what?, Museum der Kulturen Basel, Bâle.

CHRISTEN Kimberly

2009 « Opening archives: respectful repatriation », American Archivist, 74, p. 185-210.

DAVY Damien

2007 « Vannerie et vanniers ». Approche ethnologique d'une activité artisanale en Guyane française, thèse de doctorat en anthropologie sociale, université d'Orléans.

DE LARGY HEALY Jessica

2014 « Remediating sacred imagery on screens: Yolngu experiments with new media technology ", Les actes de colloques du musée du quai Branly Jacques Chirac [en ligne], 4, https:// journals.openedition.org/actesbranly/577, consulté le 10/06/2021. DOI: 10.4000/actesbranly.577 DELAîTRE Anouck et Pascale de ROBERT 2019 « De l'Amazonie brésilienne aux musées français : parcours de collections et processus de legitimation », Revista Anthropológicas, 30 (2), p. 38-62. 
ERIKSON Philippe

2019 « Avant-propos d'Itënëimëk-Kunolo », Ateliers d'anthropologie [en ligne], HS 2019, https:// journals.openedition.org/ateliers/12588, consulté le 10/06/2021. DOI : 10.4000/ateliers.12588

\section{GEERTZ Clifford}

1973 The Interpretation of Cultures. Selected Essays, Basic Books, New York.

GIBSON Laura et Rebecca KAHN

2016 " Digital Museums in the 21st century: global microphones or universal mufflers?", Museological Review, 20, p. 39-52.

GLINKA Katrin, Sebastian MEIER et Marian DÖRK

2015 "Visualising the "un-seen": towards critical approaches and strategies of inclusion in digital cultural heritage interfaces ", Kultur und Informatik, 13, p. 105-118.

GÖBEL Barbara et Gloria B. CHICOTE

2017 "Transiciones inciertas: una introducción », in Barbara Göbel et Gloria B. Chicote (dir.), Transiciones inciertas. Archivos, conocimientos y transformación digital en América Latina, Universidad Nacional de La Plata/Ibero-Amerikanisches Institut, La Plata/Berlin, p. 9-17.

HOFFMANN Beatrix et Karoline NOACK

2016 «Participation in anthropological museum research: the Apalai-Wayana and Tiriyó example ", Museumskunde. Herausgegeben vom Deutschen Museumsbund (numéro spécial : Positioning Ethnological Museums in the 21st century), 81 (1), p. 64-69.

JOLLY Eric

2008 «Le Fonds Marcel-Griaule : un objet de recherche à partager ou un patrimoine à restituer? ", Ateliers du LESC [en ligne], 32, https://journals.openedition.org/ateliers/2902, consulté le 10/06/2021. DOI : 10.4000/ateliers.2902

\section{KRAUS Michael}

2016 "The Museum as maloca? A cooperation project with indigenous partners ", Museumskunde. Herausgegeben vom Deutschen Museumsbund (numéro spécial : Positioning Ethnological Museums in the 21st century), 81 (1), p. 70-75.

\section{MAFFIE James}

2009 " "In the end, we have the Gatling gun, and they have not": Future prospects of indigenous knowledges ", Futures, 41, p. 53-65.

MBEMBE Achille

2017 « Afrocomputation. Entretien avec Bregtje van der Haak traduit par François-Ronan Dubois », Multitudes, 4, p. 198-204.

\section{NAEPELS Michel}

2008 "Contextualiser les archives missionnaires : quelques remarques méthodologiques », Ateliers du LESC [en ligne], 32, https://journals.openedition.org/ateliers/1882, consulté le 10/06/2021. DOI : 10.4000/ateliers. 1882

\section{NAGY Murielle}

2011 " Access to data and reports after completion of a research project », Études/Inuit/Studies (numéro spécial : Propriété intellectuelle et éthique), 35 (1-2), p. 201-221, https://www.erudit.org/ $\mathrm{fr} /$ revues/etudinuit/2011-v35-n1-2-etudinuit0322/1012842ar/, consulté le 10/06/2021. DOI : 10.7202/1012842ar 
PALANAIWA Akajuli, Asiwae WAYANA, Ikale ASAUKILI, Pekijen KULITAIKË et Mataliwa KULIYAMAN 2019 « Itëneimëk-Kunolo », Ateliers d'anthropologie [en ligne], HS 2019, https:// journals.openedition.org/ateliers/10883, consulté le 10/06/2021. DOI : 10.4000/ateliers.10883

PEERS Laura

2010 "Afterword », in Laura van Broekhoven, Cunera Buijs and Pieter Hovens (dir.), Sharing Knowledge \& Cultural Heritage. First Nations of the Americas. Studies in Collaboration with Indigenous Peoples from Greenland, North and South America, Leiden, National Museum of Ethnology (Mededelingen van het Rijksmuseum voor Volkenkunde, 39)/Side Stone Press, p. 187-193.

PIERREBOURG Fabienne de

À paraître «Primeras experiencias colaborativas y nuevos debates en las colecciones nacionales francesas ", in Cultures-Kairós- Revue d'anthropologie des pratiques corporelles et des arts vivants.

POWELL Timothy B.

2016 « Digital knowledge sharing: forging partnerships between scholars, archives, and indigenous communities ", Museum Anthropology Review, 10 (2), p. 66-90.

SHEPARD Jr. Glenn, Claudia Leonor LóPEZ GARCÉs, Pascale de ROBERT et Carlos Eduardo CHAVES 2017 « Objeto, sujeito, inimigo, vovô: um estudo em etnomuseologia comparada entre os Mebêngôkre-Kayapó e Baniwa do Brasil », Boletim do Museu Paraense Emílio Goeldi. Cié.incias Humanas, 12 (3), p. 765-787, https://www.scielo.br/j/bgoeldi/a/FynGr9mNDDtPWFRnsxN7shL/? lang=pt, consulté le 10/06/2021. DOI: 10.1590/1981.81222017000300006

VAN VELTHEM Lucia Hussak, Katia KUKAWKA et Lydie JOANNY

2017 « Museus, coleções etnográficas e a busca do diálogo intercultural », Boletim do Museu Paraense Emílio Goeldi. Ciê.incias Humanas, 12 (3), p. 735-748.

\section{VAPNARSKY Valentina}

2019-2020 « Des communautés sources aux communautés d'experts : l'expérience SAWA

(Savoirs Autochtones Wayana-Apalaï de Guyane) ", Culture et Recherche, Paris, p. 71-72.

\section{VAPNARSKY Valentina}

2020 "Retour aux sources ? Circulation et virtualités des savoirs amérindiens à l'ère $d u$ numérique », Journal de la Société des américanistes, 106 (2), p. 79-103.

WERE Graeme

2021 « Returned not remade: visuality, authority and potentiality of digital objects in a Melanesian society », in Paolo Fortis and Susanne Küchler (dir.), Time and Its Object. A Perspective from Amerindian and Melanesian Societies on the Temporality of Images, Routledge, London, p. $171-184$.

WRIGHT Robin, Wolfgang KAPFHAMMER et Flavio Braune WIIK

2012 " The clash of cosmographies: Indigenous societies and project collaboration - Three ethnographic cases (Kaingang, Sateré-Mawé, Baniwa) », Vibrant - Virtual Brazilian Anthropology, 9 (1), p. 384-450, http://www.vibrant.org.br/issues/v9n1/robin-m-wright-wolfgangkapfhammer-flavio-braune-wiik-the-clash-of-cosmographies/, consulté le 10/06/2021.

\section{NOTES}

1. Terminologie désignant, dans le monde informatique, les langues disposant de peu de ressources linguistiques informatisées (lexiques, correcteur orthographique, analyseur syntaxique, etc.). La création de plateformes numériques avec un accès entièrement dans 
la langue des communautés sources implique ainsi un travail très exigeant de traduction. Un article par Mataliwa Kuliyaman et Sara Tandar sur cette démarche et les néologismes wayana et apalaï créés pour la terminologie informatique et les interfaces du portails https://watau.fr devait intégrer ce dossier du JSA. Les données ayant été pré-publiées dans Camargo et al. (2020), nous renvoyons à cet article.

2. Élaboré dans le cadre du projet collaboratif Sawa (« Savoirs autochtones wayana et apalaï [Guyane]: une nouvelle approche de la restitution et ses implications sur les formes de transmission »), le portail Watau (https://watau.fr) a pour vocation de rendre accessible dans leur langue et suivant des modalités (ergonomie, visuel, classification, accessibilité...) définies avec eux un ensemble de données numérisées concernant leurs cultures. Ce projet, qui résulte d'une entreprise comparable à celle analysée dans ces pages par Scholz et Guzmán Ocampo, est à l'origine des réflexions qui ont abouti à l'élaboration du présent dossier, ainsi qu'on l'a évoqué dans l'introduction de son premier volet (Vapnarsky 2020, p. 81, n. 2).

3. Interview de Mataliwa Kuliyaman 2019, cité in Vapnarsky (2019-2020, p. 71), voir aussi Pierrebourg, à paraître. Une partie des partenaires amérindiens du projet Sawa, aidés par l'ethnolinguiste Eliane Camargo, ont rédigé un ouvrage destiné à rendre compte de leur expérience dans le cadre de ce projet (Palanaiwa et al. 2019). Bien qu'entièrement écrit en wayana à l'attention d'un public amérindien, il est accompagné d'un avant-propos en français destiné aux lecteurs moins versés dans les langues caribes (Erikson 2019).

4. Une mention de ce néologisme peut être trouvée dans Camargo et al. (2020, §31). Quoique ëwa soit généralement traduit par corde, ficelle ou cable (Camargo et Tapinkili 2021, p. 82), le terme peut également renvoyer au liber de l'écorce de certains arbres de la famille des Lécythidacées, particulièrement résistant et susceptible d'être facilement détaché en longs filaments, et utilisé pour faire des sangles. Damien Davy (2007, p. 339) qualifie ces filaments d'écorces à bretelles. Les lanières sont obtenues en entaillant l'écorce et le liber d'un arbre adéquat sur une largeur d'environ $15 \mathrm{~cm}$, pour décoller de la base un petit morceau ; celui-ci servira ensuite de poignée permettant de détacher sur plusieurs mètres, dans le sens de la hauteur, de grands lambeaux filiformes (un peu comme on arrache des lés de papier peint). Ce geste est bien sûr particulièrement propice à l'évocation, dans le registre mythologique, d'une ascension verticale (pour passer d'une strate à l'autre du cosmos). Sans doute est-ce aussi cette acception de ëwa qui explique son emploi pour traduire, dans le registre du numérique, ce que les Wayana ont entendu appeler en français « la bande passante ».

5. L'Australie fournit de nombreux exemples d'initiatives initialement destinées à la diffusion de données mais qui aboutissent paradoxalement à une situation contraire: leur mise sous séquestre au nom du respect au droit à l'auto-détermination et au secret (De Largy Healy 2014). Des situations comparables sont fréquentes en Amérique du nord, tel le cas rapporté par cette collègue constatant que: «If organisations and individuals become increasingly reticent to allow public access to interviews they originally agreed to share, archives may turn into depositories that do little to disseminate information. [In some situations, ] individuals would not object to their recordings being online but their community might. This has happened to the web-based version of Project Jukebox [developed in 1988] at the University of Alaska Fairbanks [...]. The original computer-based program was designed to integrate oral history recordings with associated texts, photographs, and maps. In recent years, however, "permission and ethical issues about making people's recordings so widely accessible have slowed the process of putting more projects online" » (Nagy 2011, p. 212). 
6. Dans d'autres cas, même si les archives restituées relèvent indéniablement du patrimoine du groupe, des problèmes peuvent se poser. Par exemple, la capacité d'exercer un rituel ayant disparu, ne reste parfois que le pouvoir de contrôler l'accès à l'enregistrement d'une performance ancienne qui s'érige donc en standard, alors qu'elle n'était au départ qu'un spécimen parmi d'autres.

7. Pour une perspective comparative avec la Mélanésie sur cette question de la différence entre l'original et la copie dans les projets de restitution, on peut se référer à Were (2021).

\section{AUTEURS}

VALENTINA VAPNARSKY

CNRS, Erea/Lesc (UMR 7186)

\section{CAMILLE NOÛS}

Laboratoire Cogitamus 